\title{
Prevalência da Associação entre DPOC e Cardiopatia Isquêmica em um Hospital do Interior do Rio Grande do Sul no ano de 2013
}

\author{
Chaves, J.; Krummenauer, M.L.; Fuhr, L.G.; Brun, D.C.; Peixoto, S.L.; Pilletti, \\ K.; Rodrigues, M.T.;
}

Apresentador: Jéssica Chaves

\section{Resumo}

Introdução: A doença pulmonar obstrutiva crônica (DPOC) é uma doença caracterizada por limitação ao fluxo aéreo não totalmente reversível, geralmente progressiva e está associada a uma resposta inflamatória anormal dos pulmões, causada primariamente pelo tabagismo. É uma doença que afeta também os vasos sanguíneos, havendo uma associação importante entre DPOC e doença cardiovascular. Métodos: Estudo de natureza descritiva de abordagem quantitativa exploratória e retrospectiva, sendo realizado através da revisão de prontuários de internação do SAME (Serviço de Arquivamento Médico e Estatístico) no Hospital Santa Cruz (HSC), em Santa Cruz do Sul. Foi analisado o perfil dos pacientes portadores de cardiopatia isquêmica internados no HSC e quais apresentavam registro de DPOC durante o período de janeiro a dezembro de 2013. A coleta de dados foi realizada no período de abril a junho de 2014. Resultados: Foram avaliados prontuários de 197 pacientes com cardiopatia isquêmica no ano de 2013. Desse total, apenas 8 (4\%) possuiam diagnóstico de DPOC registrado no prontuário. Com relação aos portadores de doença pulmonar, 4 $(50 \%)$ eram do sexo masculino e $4(50 \%)$ eram do sexo feminino, apresentando uma média de idade de 62,75 anos. Quanto ao status tabágico, 56 pacientes da amostra total $(28,42 \%)$ eram tabagistas, e 23 $(11,67 \%)$ haviam cessado tabagismo. Em relação aos pacientes com DPOC, 5 pacientes $(62,5 \%)$ são tabagistas, 2 (25\%) cessaram o tabagismo e $1(12,5 \%)$ não foi encontrado registro no prontuário. Quanto aos sintomas relatados pelos pacientes com DPOC foram encontrados registros de sintomas em apenas 2 prontuários, relatando tosse e dispneia. Quanto às comorbidades: 4 (50\%) pacientes eram hipertensos, $3(37,5 \%)$ dislipidêmicos, $2(25 \%)$ apresentavam insuficiência cardíaca e $3(37,5 \%)$ referiam IAM prévio. Conclusão: Muitos pacientes com DPOC não percebem sintomas de exacerbação já que a mesma pode mimetizar ou coexistir com outras patologias pulmonares e cardiovasculares, o que dificulta o diagnóstico dessa condição, inferindo-se que nesse estudo mais pacientes podem ter a correlação entre as doenças, porém não há registro nem diagnóstico de DPOC. O principal fator de risco em comum para as duas doenças é o tabagismo, visto neste estudo. São necessários mais estudos para determinar a real prevalência de pacientes com as duas afecções concomitantes. Nesse estudo, ressalta-se que na maioria dos pacientes os dados não foram adequadamente registrados no prontuário.

\section{Referência:}

Chaves, J.; Krummenauer, M.L.; Fuhr, L.G.; Brun, D.C.; Peixoto, S.L.; Pilletti, K.; Rodrigues, M.T.;. Prevalência da Associação entre DPOC e Cardiopatia Isquêmica em um Hospital do Interior do Rio Grande do Sul no ano de 2013. In: II Congresso Brasileiro de Medicina Hospitalar - II CBMH [= Blucher Medical Proceedings, vol.1, num.5] São Paulo: Editora Blucher, 2014. p.47

DOI 10.5151/medpro-II-cbmh-042 